\title{
Why robots should not be treated like animals
}

Deborah G. Johnson University of Virginia

Mario Verdicchio Università degli Studi di Bergamo

\begin{abstract}
Responsible Robotics is about developing robots in ways that take their social implications into account, which includes conceptually framing robots and their role in the world accurately. We are now in the process of incorporating robots into our world and we are trying to figure out what to make of them and where to put them in our conceptual, physical, economic, legal, emotional and moral world. How humans think about robots, especially humanoid social robots, which elicit complex and sometimes disconcerting reactions, is not predetermined. The animal-robot analogy is one of the most commonly used in attempting to frame interactions between humans and robots and it also tends to push in the direction of blurring the distinction between humans and machines. We argue that, despite some shared characteristics, when it comes to thinking about the moral status of humanoid robots, legal liability, and the impact of treatment of humanoid robots on how humans treat one another, analogies with animals are misleading.
\end{abstract}

\section{Introduction}

Responsible Robotics is about developing robots in ways that take their social implications into account. This involves more than just ensuring that robots are safe and used in socially beneficial ways. It also involves responsibly constructing the meaning and significance of robots, that is, framing robots and their role in the world accurately and in ways that do not lead to negative consequences. The challenge here is especially daunting when it comes to social robots, that is, robots made to interact with humans on a social level, including entertainment robots (e.g. Sony's AIBO), care bots for the elderly, and sex bots. Moreover, withinin the category of social robots, humanoid robots pose a unique challenge. Because they look and act so much like humans, they elicit complex and sometimes disconcerting human reactions, as shown in the phenomena referred to as 'the uncanny valley' (Mori, 1970; Mori et al., 2012).

An examination of the scholarly and public discourse anticipating humanoid robots suggests that there is contestation over the meaning and significance of such robots. In general there is controversy as to whether robots will be a panacea or a bane. Many contemplate, for example, whether robots will radically reduce the need for human labor (Ford, 2015) or fundamentally change the nature of warfare (Future of Life Institute, 2015). When it comes to humanoid robots, the discourse is more speculative but nevertheless controversial. Some argue that humanoid robots will become so human-like in appearance and capability that they will have to be granted something comparable to human rights (Robertson, 2014). Others argue that this never should or will happen and that robots should intentionally be built in ways that make it clear that they are not human (Miller, 2010; Grodzinsky et. al., 2015). Yet others see humanoid robots as potentially the ultimate replacement for human companionship (Hauskeller, 2016). Speculation here is that some humans will in the future choose humanoid robots over humans for their life partners (Levy, 2008). 
Whether it is robots in general or social robots or humanoid robots in particular, robots are an evolving/emerging technology and along side the material design and operation of new technologies come social ideas about what they are and where and how they fit into the human world. We are now in the process of incorporating robots into our world and we are trying to figure out what to make of them and where to put them in our conceptual, physical, economic, legal, emotional and moral world. But we are doing this while we are simultaneously making them. It is not that we have discovered a new entity and are poking and prodding it to figure out what it is. Rather we are at once creating a new type of entity and simultaneously asking what it is. This process is especially complicated in the case of humanoid robots because of their human-like characteristics and the trend towards making them more and more humanlike (Glas et al., 2016; Hanson, 2017).

Because robot technology is still evolving, there is an opportunity for reflexivity. That is, there is an opportunity to step back and think about how robots might impact human society and human values and an opportunity to conscientiously intervene in the process (Chilvers, 2013; Owen et al., 2013). How humans think about robots, especially humanoid social robots, is not predetermined. The process is contingent and there are advantages and disadvantages of going one way or another. Reflection on the process of assimilation while it is taking place and self-consciously trying to shape what is made of such robots has the potential to help ensure that robots of the future will be more socially beneficial.

The reflexive process is daunting and in this paper, we will only take a small step in that direction. Our interest is in how analogies with animals are being used to try to understand humanoid robots. To be sure, analogies with animals are being used more broadly to conceptualize social robots (robot designers do this explicitly when they design robots to look like pets); however, our concern here is primarily with humanoid social robots. Although much of our analysis can be applied to robots in general and to non-humanoid social robots, we restrict our analysis to this unique kind of robot. We do so because robots that look like humans and interact with humans socially have the potential to radically challenge what it means to be human.

Although roboticists and others will know how robots work and how they are different from humans, the appearance and behaviour of humanoid robots will call upon humans to think and feel that humanoid robots are human or at least some new species of human. While some may not find this problematic, others will, and, in any case, this possibility seems worthy of some deep reflection.

We tackle the animal-robot analogy because it is one of the most commonly used in attempting to frame interactions between humans and robots and it also tends to push in the direction of blurring the distinction between humans and machines. In section 2 we explore the many reasons why it is tempting to use animals as a model for assimilating humanoid robots into the human world. Nevertheless, our aim is to challenge that temptation and to argue that it is misleading to use analogies with animals as the basis for our conceptual understanding of humanoid robots. We do this by examining how analogies with animals are used in thinking about the moral status of humanoid robots, legal liability for robot behavior, and the impact of treatment of humanoid robots on how humans treat one another. 
It may be helpful to note in advance, that it is not surprising that scholars and lay thinkers make use of analogies to think about robots. Analogical reasoning is a common strategy for understanding new phenomena. We use something familiar to understand something unfamiliar or less familiar. Gentner and Forbus (2011) explain that in typical analogies, a "base (or source) domain is mapped to a less familiar (or more abstract) target domain." Gentner and Forbus point out that the inference from base to target is selective. We select certain characteristics of the base or target and not others. Just how or why selections are made is a matter of empirical research and a subject of study among psychologists, cognitive scientists, and others (Holyoak and Koh, 1987; Ross, 1989; Spellman and Holyoak, 1996).

In the analysis that follows, analogies between animals and robots will be shown to involve the selection of such characteristics as otherness, capacity to trigger humans to anthropomorphize and attach, trainability, and potential to assist humans and harm humans. Our argument is that despite these selected, shared characteristics, when it comes to thinking about the moral status of humanoid robots, legal liability, and the impact of treatment of humanoid robots on how humans treat one another, analogies with animals are misleading. They neglect the fundamental difference between animals and robots, that animals suffer and robots do not.

\section{The Inclination to Use Analogies with Animals}

We begin our analysis by acknowledging many of commonalities that tempt us to use ideas about animals to think about robots. Many of them apply to robots in general but several are especially compelling in the case of humanoid robots.

Coeckelbergh (2010) suggests that robots and animals are alike insofar as both are not human. They both are 'others' in relation to humans. In other words, animal-human and robot-human relationships are both alterity relationships. Importantly, both animals and robots are thought to be not just not human, but less than human. Animals and robots are generally seen as lacking in some respect by comparison to humans; they have less value and less or no moral status.

Coeckelbergh is not focused on how we should understand robots per se; he is focused on relationships, namely human to other relationships. What seems to matter for Coeckelbergh is how robots appear to us. His position emphasizes how humans experience robots and animals, and that depends on their appearance. In this respect, his argument seems implicitly to be a version of an argument that we will take up later, namely that what is important about how we treat robots (and animals) is how such treatment ultimately affects how we treat one another, i.e., other humans. This argument is especially compelling in the case of humanoid social robots in that these robots are closer in appearance to humans than non-human or non-social robots. If robot-human relationships affect human-human relationships, the effect of humanoid social robot-human relationships are likely to be the strongest.

The subordinate dimension of the otherness of animals and robots is emphasized by other authors. Ashrafian (2015) writes that: "Non-human animals species are subordinate to mankind and in a similar fashion robots and artificially intelligent agents will also be subordinate to humans." Here status seems to be related to the 
historical idea of a hierarchical chain of being with humans at the top and animals lower down but there are several strains of thinking that have supported the lower status of animals (Calverley, 2006). On this line of reasoning, since robots will also be subordinate, animals are an appropriate model. That is, they are a model for how we treat things that are less than human but still have characteristics or features that give them some sort of moral status.

Darling (2016) uses the animal-robot analogy in precisely this way when she writes:

Animals themselves are not protected from being put down, but rather only when ending their lives is deemed cruel and unnecessary given the method or circumstances. Similarly, it would make little sense to give robots a «right to life.»

The otherness of animals and robots has led scholars such as Gunkel (2012) and Hogan (2017) to ask whether the 'machine question', i.e. are machines legitimate moral agents?, is the same as the 'animal question'. Gunkel's analysis goes to great lengths to go beyond ontological issues with regard to machines and animals and elaborates on the concept of otherness in an attempt to establish a significant moral status for machines (Gunkel, 2014, 2017). On the other hand, Hogan recognises the necessity to acknowledge biological evolution in a discourse on morality in which she claims that the two questions are different and suggests a more human-centric reformulation of the machine question. We will not evaluate their analyses, but Hogan seems to acknowledge that mere otherness is not sufficient to justify using one as a model for the other.

In addition to sharing subordinate otherness, both animals and robots have the capacity to elicit responses in humans, responses that are characterized as anthropomorphization and attachment. Although no scholars that we know of have explicitly used this as the basis of an animal-robot analogy, this attribute of humanoid robots shared with animals seems to influence many approaches to understanding the significance of humanoid robots. We attribute both to animals and to robots qualities, feelings, and states of being that we (humans) have. The anthropomorphization of certain animals is commonplace and the anthropomoraphization of humanoid social robots is intentionally designed to have this effect.

Whatever the mechanisms by which anthropomorphization occurs, it can lead to (or at least contribute to) humans developing attachments to both animals and robots (Chin et al., 2004; Epley et al., 2007; Fussell et al., 2008; Eyssel et al., 2012). Intense, even extreme, versions of such attachments in the case of humanoid robots have often been explored in science fiction (Dick, 1968; Moore, 1989; Asimov, 1993; Gibson, 1996; Jonze, 2013; Garland, 2015).

There seems little doubt that humans anthropomorphize and attach both to (some) animals and (some) robots. Still, as we will argue, whether this capacity to elicit anthropomorphization and attachment is sufficient to justify using one type of entity as a model for treatment of the other is quite a different matter.

Related to anthropomorization and attachment, animals and robots both have the capacity to assist and support humans, even in very intimate ways. Both have the 
capacity to do labor for humans - labor that humans can't or don't want to do. Think here of industrial robots as a modern version of oxen moving the simple, mechanical parts of a grain mill. In more intimate roles, pets serve as companions to humans, guide dogs assist the blind, and guard dogs protect their owners. Robots are envisioned to assist humans in a variety of ways and humanoid social robots are an especially compelling case since they have the potential to serve as caregivers for the elderly (Borenstein, 2010; Sharkey, 2012), nannies for children (Sharkey, 2010), and even sex partners (Sharkey et al., 2017).

Sullins (2011) brings animals into parallel with robots by arguing that domesticated animals such as guide dogs are a technology and, in this respect, are like robots. As technologies, both guide dogs and robots consist of a similar triad of parts; trainer, guide dog, and user (e.g., a blind person) and programmer, robot, and user (e.g., a customer). On the basis of this parallel, Sullins argues that it makes sense to think of domesticated dogs and robots analogously:

For millennia humans have been breading[sic] dogs for human uses and if we think of technology as a manipulation of nature to human ends, we can comfortably call domesticated dogs a technology. This technology is naturally intelligent and probably has some sort of consciousness as well, furthermore dogs can be trained to do our bidding, and in these ways, dogs are much like the robots we are striving to create. (p. 24)

Ashrafian (2015) makes a similar move to emphasize the shared role of animals and robots in fulfilling human purposes. He writes:

Many animals are reared by humans to fulfill specific duties in human society (such as guide dogs). In many cases they are also specifically bred (with defined genotypes, phenotypes and traits) and subsequently trained for specific tasks. In a similar way robots and artificially intelligent agents are specifically designed, built and subsequently programmed for specific tasks.

Of course, this use of the animal-technology analogy is tricky because it could be used in the opposite direction, that is, it could be used as a reason to see and treat animals like machines as Descartes did.

We do not dispute any of these commonalities between animals and robots. Our argument is that none of them is sufficient to justify using attitudes and practices with regard to animals as a model for understanding and treating robots, especially humanoid robots. Moreover, we are concerned that the use of animal analogies is misleading in the context of discussions of moral status, legal liability, and the impact of treatment on how we treat one another.

\section{Why Animal-Robot Analogies Fail}

Our concern that animal analogies are misleading is connected to robots being in the early stages of development when there is uncertainty about how to understand and treat them. As explained earlier, the meaning and significance of robots is being 
socially negotiated and this is especially complicated in the case of humanoid social robots. The negotiation is most evident in discussions of moral status, legal liability, and impact on the treatment of humans by humans. Our argument is that in these important contexts the fundamental difference between animals and all robots (humanoid, social and otherwhise) should not be misrepresented. Animals are living organisms and robots are not. Robots are machines. Animals are sentient organisms, that is, they are capable of perception and they feel, whereas robots do not, at least not in the important sense in which animals do ${ }^{1}$.

We recognize that there is an enormous variation among non-human animals and in human thinking, norms, and laws with respect to different animals, especially between those that are domesticated as pets and those that are wild. Our argument is focused on analogies with animals that suffer, and although we recognize that there is variation in the degree to which different animals suffer, we do not take up this matter since our argument against animal-robot analogies rests simply on the claim that robots do not suffer.

When it comes to discussion of the moral status, legal liability, and the impact on human-human treatment, analogies with animals are misleading.

\subsection{Moral Status}

In discussions of the moral status of robots, animal-robot analogies come into play in arguments about whether humanoid social robots of the future might be granted rights. The claim is that humanoid robots of the future will have qualities or characteristics that will justify granting them rights, possibly even the right to life (McNally and Inayatullah, 1988; Spennemann, 2007; Anderson and Anderson, 2011; Lin et al., 2011; Parisi, 2014). A discussion of robot rights has already been started in the European Parliament, whose Committee on Legal Affairs drafted a document in which a possible future is envisioned in which more and more autonomous robots become so sophisticated that they should be established "as having the status of electronic persons with specific rights" (Delvaux, 2016).

Many turn to animals as a model because animals have legal and moral status which effectively recognizes rights ${ }^{2}$. There are moral and legal rules about what can't or shouldn't be done to animals. Thus, it is worth considering what robots and animals have in common and whether analogies with animals make sense. Are animals an appropriate model for thinking about the moral status of robots?

\footnotetext{
${ }^{1}$ Many roboticists talk about robots "feeling" or "sensing" the environment because these machines are endowed with sensors, but their discourse is metaphorical.

${ }^{2}$ Some scholars, like Solaiman, turn to animals as a model to deny that robots should be granted personhood (Solaiman, 2017). The scholar uses a case in which a judge denied personhood to chimpanzees to argue against the idea of conferring legal personhood to robots. This all-or-nothing approach on personhood (either animals and robots have all the rights and duties connected with personhood or they don't have any) may be too coarse-grained for our analysis, since it begs the question on why there are laws against animal cruelty even though animals are not considered persons.
} 
For the most part, discussion of moral status in relation to futuristic robots has focused on their potential sentience or their mental capabilities e.g., autonomy, consciousness. The discussion is less concerned with the significance of human-like appearance. In this context, the animal-robot analogy is used explicitly and implicitly, and often by way of suggesting that since animals acquire moral status from their capacity to suffer, if robots have the capacity to suffer, they too would have to be given moral status. The problem is that robots do not suffer and even those of the future will not suffer. Yes, future robots might have some states of being that could be equated with suffering (Rysewyk, 2014; Kuehn and Haddadin, 2017) but, futuristic thinking leaves it unclear what - other than metaphorical representation - it could mean to say that a robot suffers. Thus, the animal-robot analogy doesn't work here. Animals are sentient beings and robots are not.

As an aside here, even if we remain open to the possibility of building robots that suffer, and before jumping to the conclusion that such entitities would have moral standing, some have questioned the wisdom of building something that can suffer (MacLennan, 2013; Metzinger, 2013). Wouldn't it be wrong to build robots that suffer? It would be bringing more suffering into the world.

Consciousness is another dimension of sentience that comes into play in discussions of the moral standing of animals and robots. In the literature on futuristic robots, consciousness is often discussed as a potential feature of robots (Levy, 2009; Ramey, 2005; Calverley, 2005, 2006). Some argue that some animals have something like it and/or that although robots do not have it now, they may have it in the future. In one of the more interesting takes on this, Levy (2009) assumes androids will be conscious in the future but he disconnects consciousness and suffering arguing that even with consciousness, androids will not suffer. Levy's position is even more complex in that he argues for robot rights. He argues that neither consciousness nor suffering are the relevant characteristic when it comes to rights. Rather, it is the effect of our treatment of robots on how we treat one another that makes the case for giving robots rights. This argument, which has been made by others in addition to Levy, will be explored further in section 3.3.

Levy's position is an imaginative leap. The possibility of conscious robots that are not capable of feeling pain raises many difficult questions. Firstly, what does it mean to be conscious and yet not able to suffer? Does suffering refer only to physical pain or does it include also mental suffering, such as boredom, loneliness, and guilt? In particular, could we consider humanoids that do not feel guilt to be moral agents? On the other hand, would it be moral for us to build a robot that can get bored and lonely while attending to its repetitive tasks? Again, this would involve bringing more suffering into the world.

In any case, if sentience, suffering, and consciousness are used as the basis for moral status, the analogy between animals and robots doesn't work. Robots of today do not have sentience or consciousness and do not suffer. Robots of the future might have characteristics that are equated with sentience, suffering, and consciousness, but if these features are going to be independent of each other as imagined by Levy, or even recommended by Metzinger (2013) to ensure that robots will not suffer, they will be fundamentally different from what humans and (some) animals have. It is the 
capacity to suffer that drives a wedge between animals and robots when it comes to moral status.

Some of the literature on the moral status of robots focuses on the moral agency of future robots. For example, according to Sullins, one of the scholars focused on programming robots to adhere to moral rules and principles, a time may arrive when rights will have to be extended to robots because they are entities capable of moral evaluations, decisions, and actions (Sullins, 2006). Sullins does not here make an analogy with animals and it is not surprising that he doesn't for when it comes to the agency of animals, there is little consensus or foundation. There is little understanding or agreement on whether any or which animals can be said to be agents of their actions. When are animals "responsible" for their behaviour? In the next section on legal liability we will see that when it comes to blame and responsibility the focus of attention is not on the agency of the animal (or the robot) but on the owner.

Importantly, when it comes to moral status, there is a difference between moral agents and moral patients. Moral patients derive their moral status from their capacity to suffer and be harmed while moral agents derive their moral status from their capacity to act and to cause suffering and harm. So, when agency is used as the basis for the moral status of robots, analogies with animals don't work. Animals suffer and therefore have a right to protection from those who might harm them. Those who argue for futuristic robots as moral agents (and for this as the basis for assigning rights to robots) argue for this on the basis of what robots will be able to do (especially when they are programmed to behave morally). To be sure, as already indicated, some argue that we might have to grant moral standing to robots of the future if they have the capacity to suffer. Such arguments are, indeed, patient-based but we have already shown the problems with such arguments. In any case, animalrobot analogies focused on moral agency do not work because animals acquire moral standing as moral patients not moral agents.

Interestingly, Gunkel (2014) explores the agent-patient distinction in making the case for the rights of machines. Arguing that the move to acknowledge moral standing to entities who experience pain and suffering (patients) constituted a revolution in ethics, Gunkel attempts to bring animals and machines in line as patients. However, in the end he rejects the undertaking: "we cannot, it seems, make a credible case for or against the moral standing of the machine by simply following the patient-oriented approach modeled by animal rights philosophy" (p. 121). Instead Gunkel takes the failure as a sign that the revolution in ethics is suspect and that there are "systemic problems in the very structure and protocols of moral reasoning" (p. 122). This move leaves animal-robot analogies no where, and rejecting the structure and protocols of moral reasoning seems to leave us entirely adrift in understanding the moral status of any entity.

Somewhat related to the focus on agency, some of the literature on the status of robots focuses on legal personhood. For example, although Bryson et al. (2017) avoid futuristic considerations of robots that might be conscious or have something comparable to pain, they review cases involving the conferral of legal personhood. Arguably, legal personhood might be thought of as agency. Bryson et al. consider whether precedents for legal personhood can be adapted to tackle the issue of legal 
liability of machines and they conclude that legal personhood should not be used for artificial entities. In any case, Bryson et al and the discourse on legal personhood of artificial entities tends not to use analogies with animals since animals are not considered legal persons.

So, when it comes to the moral status of robots generally and humanoid social robots in particular, analogies with animals seem not to provide any substantive ground for inferring how robots ought to be understood and treated. Yes, some robots and animals share characteristics; however, these shared characteristics do not justify using animals in thinking about the moral status of robots because animals derive their moral standing from their sentience and their capacity to suffer and robots are machines and do not suffer.

There is one possible exception to this. As already suggested, some argue that the capacity of an entity to affect how humans treat one another has relevance to the entity's moral status. This claim needs to be carefully examined and we will do so in section 3.3.

\subsection{Legal liability and assignment of responsibility for robots}

As part of the process of understanding and incorporating humanoid robots into society, one of the issues that has been raised has to do with who will be responsible for the harmful behaviour of robots. Both animals and robots have the potential to behave in ways that are harmful to humans, e.g., physically attacking, failing to do what is expected, or causing harm in some other way. Legal liability for animal behaviour has been suggested as a model for robot liability. When it comes to law, animals are not considered themselves to be liable for their behaviour; rather, owners are legally liable. Although some have suggested that futuristic robots might someday be held responsible for their own behaviour, the discourse on legal liability using animal-robot analogies does not point in that direction. Rather the analogy with animals is used to identify the conditions under which manufacturers and owners of robots might be held liable for robot behaviour.

Asaro (2012) suggests that using animals as a model for robots has the advantage that it does not require that we give any special rights or considerations to robots, we need only be concerned with their owners (p. 178). Both domesticated animals and robots are property, so liability can be dealt with through property owners. With this in mind, then, it would seem to make sense to look at the liability of animal owners as a model for addressing liability in the case of robots.

One set of authors has done just this. Schaerer et al. (2009) argue for an approach that they call the 'Robots as Animals' framework. Their analysis draws on the legal concepts of strict liability and negligence. ${ }^{3}$ Importantly, strict liability is used for wild animal pets and negligence for domesticated pets. They argue that semi-autonomous

\footnotetext{
${ }^{3}$ Strict liability means liability does not depend on intent to do harm or negligence. With strict liability, one is liable regardless of the fact that one had no ill intent and may have taken precautions to prevent the harm. By contrast, negligence involves failure to take proper care in doing something.
} 
machines are more analogous to domesticated animals because their behaviour is more predictable and can be programmed. Hence, the conditions for negligence in the case of animal owners should be looked at for understanding negligence in the case of robot owners. Because the behaviour of wild animal pets is less predictable than that of domesticated animals, the law compels those who take on the 'extraordinary risk' of wild animals to bear the consequences when accidents occur (p. 75). Hence, Schaerer et al. conclude that:

"The owner of a semi-autonomous machine should be held liable for the negligent supervision of that machine, much like the owner of a domesticated animal is held liable for the negligent supervision of that animal. Semi-autonomous machines, like domesticated animals, are more predictable than wild animals, but remain occasionally prone to sporadic behavior - even in the absence of manufacture or design defects, and even despite adequate warnings ..." (p. 75)

The 'Robots as Animals' framework draws attention to the ability of robot manufacturers and owners to control the behaviour of the robot. In both cases (i.e., domesticated animals and robots), the owner (and/or manufacturer in the case of robots) can control the behaviour by programming and training. ${ }^{4}$ Thus, according to Schaerer et al., the focus of the law should be on whether robot manufacturers or owners (who might change the operation of a robot through training programs) were negligent in their training and monitoring of a robot.

Applying the concepts of strict liability and negligence to robots makes sense especially since these concepts are used in the treatment of other manufactured products. The question is whether the analogy with animals adds anything. After all, these legal concepts can be used as they apply to manufactured projects without bringing in animals or bringing animals in as only one among many precedents when strict liability and negligence apply.

Schaerer et al. suggest that animals are the appropriate model because both domesticated animals and semi-autonomous robots have some degree of autonomy. The problem is that when autonomy and unpredictability are attributed to animals and robots, the terms are being used in crude, metaphorical ways. Whatever autonomy is, robot autonomy and animal autonomy are radically different. Likewise, the mechanisms that make robots unpredictable and those that make animals unpredictable are radically different. These differences in turn mean that training is different in each case. In the case of domesticated animals, training operates on a natural, living entity; when it comes to robots, training operates on a human created invention and in controlled conditions. An animal trainer must work with the nature of a particular kind of animal while a robot trainer may work with a particular machine but that machine was created by other human beings who are responsible for

\footnotetext{
${ }^{4}$ In a later paper, Kelley et al. (2010) further modify the Robots as Animals framework by specifying that the important distinction is between robots that are dangerous and robots that are safe. Using an analogy with dangerous dogs, they suggest that bans or restrictions might be appropriate for dangerous robots.
} 
the nature of the machine. Thus, the analogy with animals obfuscates rather than illuminates understanding of robot liabilty.

Asaro (2016) criticizes the "Robots as Animals' framework by suggesting the difficulty of determining whether an advanced AI or robot is appropriately domesticated and will stay that way. He points out that a "designated liable person may not know the extent to which the autonomous system is capable of changing itself once it is activated" (p. 193). He also worries that a system of strict liability might result in the slow adoption of beneficial AI technologies because of the risks associated with strict liability law. In effect, Asaro suggests that the analogy with animals is not nearly as illuminating as Schaerer et. al. would have us believe.

Because robots are created by humans, human beings are not just owners, they are also manufacturers (robots are produced by designers, factory workers, investors, etc.). There are no manufacturers per se in the case of animals. No one, we might say, is responsible for nature. Of course, some might argue that breeders are responsible for the existence of certain kinds of animals, e.g., pit bulls, lab rats, and that genetic scientists are responsible for dangerous genetically engineered organisms, but here it seems the analogy justifes using robots or other manufactured goods as a model for addressing issues around animals - bred animals - than an occasion for using animals to help understand robots.

Even when animals are engineered (bred), the engineering is done on a natural object and this fact is relevant to the degree of control that humans have over the outcome. Humans have much more control over the behavior of robots since they are entirely created by humans. Humans can exert some control over the characteristics of animals by means of breeding: some desirable traits can be selected and bred through the preferential mating of individuals that display those traits. The discovery of the phenotype-genotype relation has obviously facilitated the process, and several DNA tests are available, for instance in dog breeding, to enable selection for or against specific mutations, including those that are not immediately observable, like a tendency to develop certain kinds of disease. However, knowledge of genetics is not developed enough to deal with more complex traits that are presumably based on the interaction of dozens or even hundreds of genes, possibly including the animal's character and disposition to training.

Trying to pin down the degree to which animals can be trained would take us deeply into the nature-nurture debate. More likely our point here will be challenged by some on grounds that autonomous robots (of today and even more so in the future), just like animals, will not be under the full control of the humans who created them. However, no matter what autonomy is in robots, the robots will have been created entirely by humans. Differently from what happens in genetics, humans do have a complete knowledge of the workings of the electronic circuitry of which a robot's hardware is comprised, and the instructions that constitute the robot's software have been written by a team of human coders. Even the most sophisticated artefacts that are able to learn and perfect new tasks, thanks to the latest machine learning techniques, depend heavily on human designers for their initial set-up, and human trainers for their learning process (Levine et al., 2016). Ultimately, humans bear responsibility and liability for what they create and put into the marketplace where they have effects on other humans (Johnson and Verdicchio, 2017). 
So, although parallels between the legal liability of animal owners and robot owners can be identified, the parallels involve the use of legal concepts that can be applied to robots regardless of how they are applied to animals. The concepts of strict liability and negligence seem relevant to legal liability for robot behaviour but not because robots are like domesticated animals, but simply because they are manufactured products with some degree of unpredictability. The fundamental difference between animals and robots - that one is a living organism and the other a machine - makes analogies suspect in the context of liability because liability depends on the capacity of a manufacturer or owner to control the behaviour of its property. In the case of animals, owners exert their influence through training of a natural entity; in the case of robots, manufacturers exert their influence in the creation of robots and they or others (those who buy the robots) may also exert influence via training. For this, animals are not a good model.

\subsection{Impact on social behaviour: How we treat robots and each other}

In both of the contexts just discussed - moral status and legal liability - reference is often made to the effects of our treatment of animals and robots on our own moral character and especially on how we treat one another. Kant is famous for claiming that cruelty to animals leads to cruelty to humans (Kant, 1997). Darling (2016) extends the Kantian argument to robots:

The Kantian philosophical argument for preventing cruelty to animals is that our actions towards non-humans reflect our morality - if we treat animals in inhumane ways, we become inhumane persons. This logically extends to the treatment of robotic companions. Granting them protection may reinforce behavior in ourselves that we generally regard as morally correct, or at least behavior that makes our cohabitation more agreeable. It may also prevent desensitization towards actual living creatures and protect the empathy we have for each other. (p. 19)

Darling's argument includes at least three different claims. The first is that if we treat robots (as with animals), in inhumane ways, we become inhumane persons. The second is that our treatment of robots (as with animals) may reinforce human behaviour that is morally correct or incorrect. And, the third is that treating robots (as with animals) in cruel ways may desensitize us to cruelty when it comes to people and undermine the empathy that we have for one another. The thrust of all three claims is that granting protection to robots from certain acts by humans might be justified on grounds that our treatment of robots will affect our behaviour towards one another.

There are two problems with extending these Kantian claims to robots. The first has to do with the lack of evidence to support the argument in the case of animals and the second has to do with extending the argument to robots. First, however, it is important to note that in the case of animals, there is a connection between cruelty and suffering. Cruelty involves the infliction of suffering; it involves causing pain and distress. Yes, there is more to it than that for cruelty entails the intention to do harm and the intent must be malicious. Doctors sometimes inflict pain and distress intentionally but they do so for beneficent reasons, i.e., not maliciously; they do this 
to help their patients. Hence, doctors are not considered cruel. Nevertheless, although cruelty involves more than suffering, suffering is essential to it.

The Kantian argument with regard to animals is that engaging in and/or witnessing the intentional and malicious infliction of suffering in animals may reinforce our tolerance of, and desensitize our reactions to, such cruelty to humans. The problem with this argument is that we don't know if it is true. That is, we don't know whether there is such a carryover effect or, if there is, how powerful it is. Although our intuitions sometimes go with Kant as, for example, when we witness a person treating an animal cruelly and we suspect the person must treat his children and wife cruelly as well. We do this, however, with no concrete evidence to support the inference and sometimes admit that the inference is unfair. We also have experiences that suggest the inference is mistaken; for example, we know individuals that slaughter animals (on their farms or in the meat packing industry) who are not more prone to cruelty than others; and we know hunters who despite their intentional killing of animals do not seem prone to cruelty in their human relationships.

The argument regarding animal cruelty is not, then, a strong one, and it becomes even weaker when extended to robots because while animals actually suffer in acts of cruelty against them, robots do not. The sight of a person being cruel to a robot is a simulation of cruelty. The person acting against the robot may intentionally and maliciously (say, in a fit of frustration) stomp on a robot and smash it to bits, but in doing so the person will not be causing pain and suffering. The stomper destroys property and in that sense is doing something harmful, but the stomper is not inflicting suffering. Robots do not experience pain and distress. Since robots do not suffer, when humans smash them, it is inappropriate to characterize their behaviour as cruel (though the behaviour may be far from admirable).

So, our argument here is the same as in the case of moral status. Using animals as a model for thinking about the treatment of robots is flawed because robots are not sentient and do not suffer. In affirming this, however, we are not claiming that the way we treat robots will have no impact on how we treat one another. Rather, we are claiming that our practices with regard to animals are not helpful here. They point us in the wrong direction.

To put this more directly, we ought to be concerned about how our treatment of robots affects our treatment of one another. Its just that animals are not exactly relevant to this question since our treatment of animals involves suffering. In the case of robots, what is at stake is the appearance of suffering, not suffering itself. Here again the question is most compelling in the case of humanoid social robots for the appearance of suffering can be all that more intense and believeable when one sees a seemingly human figure show signs of pain and suffering; the experience may be even more intense if one has an ongoing social relationship with the machine showing signs of suffering.

As an aside here, it is worth asking why robot designers would want to build robots that shriek and cry and take defensive positions when they are attacked? Robot builders are generally interested in building robots that work well with humans. They also have an interest in not having their robots destroyed. If building robots to appear 
to suffer when they are attacked or damaged results in better service or less damage to robots, then perhaps robot builders will be motivated to simulate suffering.

We can hypothesise that humanoid robots have the capacity to elicit emotional responses in humans from our experiences of current robots and also from our experiences with other simulations of human cruelty and suffering as in the case of visual media, e.g., photographs, films, virtual environments. Our emotions are triggered when human suffering is depicted in photographs and when we see violence in films. It is the same with more pleasant emotions evoked by photographs and films.

One of the unknowns here - one that is highly relevant to robot violence - is whether knowledge that the act is a simulation eliminates or diminishes carryover effects from simulated cruelty to cruelty towards humans. Such knowledge seems to have an effect in the case of violent movies where it seems that viewers exposed to cruelty and suffering in a film do not carry it over to their real lives. However, the effects are controversial: some believe intuitively that viewing violent movies hardens us to the sight of violence and cruelty in reality; others believe the carryover effect is stopped by the cognition that it is 'just a movie'. There are a number of experiments in favor of the former belief, suggesting that viewing a violent movie provokes higher levels of hostility (Anderson, 1997) or reduces aid offered to people in pain (Bushman and Anderson, 2009). However, time series analyses between the years 1960 and 2012 seem to show that violent films are negatively, although non-significantly, related to homicides and aggravated assaults (Markey et al., 2014).

So, this is an unresolved issue though it is an issue that ultimately could affect how robots are designed. One question this raises is whether humans should always know when they are interacting with a robot, not a person (Miller, 2010; Grodzinsky et. al., 2015). For now, humanoid robots are such that it is obvious that they are not human so our experience of them is much like that with film characters who we know are not real. However, if humanoid robots become indistinguishable in appearance from humans, then the effect of witnessing cruelty done to a human and done to a humanoid robot may be the same.

This raises an interesting question as to whether there should be prohibitions against building robots without indicators that they are robots. ${ }^{5}$ The problem is that there may be some contexts in which robots will provide better service if they look completely human, for example, in caregiving roles. So, it is not a simple question to decide whether humanoid robots should always be identifiable as robots. The important point here is that whether or not or how our treatment of robots might affect our treatment of one another may depend on whether we know the entity being treated with cruelty and appearing to suffer is a robot or not. The effect may also be different depending on the context.

${ }^{5}$ Currently there are a number of codes or standards for robotics such as the EPSRC Principles of Robotics that have a thrust in this direction but are not specific. For example, the $4^{\text {th }}$ rule in EPSRC's Principles for Designers, Builders, and Users of Robots is that: "Robots are manufactured artefacts. They should not be designed in a deceptive way to exploit vulnerable users; instead their machine nature should be transparent." (EPSRC, 2010). 
In any case, although we don't know the answer to the question whether our treatment of robots, especially humanoid robots, will affect our treatment of one another, using animals as an analogous case points us in the wrong direction. It points us to suffering but in the case of animals, there is suffering, and in the case of robots there is merely the appearance of suffering, not the reality. Yes, both involve the appearance of suffering but when it comes to robots, the appearance is a deception and, most importantly, one that does not have to occur. Robot designers can avoid the appearance of suffering in robots.

If humanoid robots of the future will look much more like humans than do animals, we would do better to look at how engaging in and witnessing cruelty towards humans affects our treatment of humans in other contexts. We have already suggested cruelty and violence as depicted in films is one place to look. Another important place to look is the carryover effects of war time experiences. Soldiers engage in and witness human beings being treated cruelly; they engage in and witness human suffering resulting from their own or others' intentional and malicious acts of cruelty to human beings. The attention now being given to Post Traumatic Stress Disorder (PTSD) (Elbogen et al., 2014; MacManus et al., 2015; Novaco et al., 2015) might be a fruitful site for exploring how the sight of cruelty and suffering in humans (and humanoid robots) might affect how humans treat one another.

This much seems clear: if it were found to be true that the sight of cruelty to humanoid robots desensitized us to the sight of cruelty in humans or that engaging in cruelty to humanoid robots increased the likelihood that we would be cruel to one another, this would provide some justification for action. The justified action could but need not necessarily be to grant rights to robots. There are at least two different directions that might be taken. One would be to restrict what could be done to humanoid robots and the other would be to restrict the design of robots. For the latter, there are several possibilities; for example, restrictions might be put on the design of humanoid robots so they don't look too humanoid or there might be a requirement that all humanoid robots display an indicator that they are a robot. Whether it be rules for treatment of robots or rules for their design, we should keep in mind that this would not be unusal. After all, we have lots of rules regarding the use of other machines.

\section{Conclusion}

The aim of this paper was to make a contribution to the big question about how we should responsibly understand (conceptualize, think about, frame) and treat robots and especially humanoid social robots. By asking a smaller question about the appropriate use of analogies with animals, our analysis has shown that in three contexts in which understanding of robots is being socially negotiated - moral status, legal liability and the impact on how humans treat one another, analogies with animals are not helpful and are misleading. Discussion in these three contexts suggests that analogies with animals do not provide a useful approach to understanding what robots are. Whatever way robots in general and especially humanoid social robots are socially and materially constituted, that is, whatever conceptual, material, legal, economic, societal and ultimately existential notions are 
developed, robots should not be understood to be something they are not. Hence, responsible discourse on robots should be cautious in using analogies with animals.

\section{References}

Anderson, Craig A. Effects of Violent Movies and Trait Hostility on Hostile Feelings and Aggressive Thoughts. Aggressive Behavior 23:161-178, 1997.

Anderson, Michael and Susan L. Anderson (editors). Machine Ethics, Cambridge University Press, 2011.

Asaro, Peter M. The liability problem for autonomous artificial agents. Ethical and Moral Considerations in Non-Human Agents, 2016 AAAI Spring Symposium Series. 2016.

Asaro, Peter M. A Body to Kick, but Still No Soul to Damn: Legal Perspectives on Robotics. In Patrick Lin, Keith Abney and George A. Bekey (eds) Robot ethics: The ethical and social implications of robotics, MIT Press, Cambridge, 2012.

Asimov, Isaac. Forward the Foundation, Doubleday, 1993.

Barrat, James. Our Final Invention: Artificial Intelligence and the End of the Human Era, Thomas Dunne Books, 2013.

Bijker, Wieber E., Thomas P. Hughes, Trevor Pinch and Deborah G. Douglas. The Social Construction of Technological Systems: New Directions in the Sociology and History of Technology, MIT Press, Cambridge, 2012.

Borenstein, Jason, and Yvette Pearson. Robot caregivers: harbingers of expanded freedom for all? Ethics and Information Technology 12(3):277-288, 2010.

Bryson, Joanna J., Mihailis E. Diamantis, Thomas D. Grant. Of, for, and by the people: the legal lacuna of synthetic persons. Artificial Intelligence and Law, 25:273291, 2017.

Bushman, Brad J. and Craig A. Anderson. Comfortably Numb: Desensitizing Effects of Violent Media on Helping Others. Psychological Science 20(3):273-277, 2009.

Calverley, David J. Android science and animal rights, does an analogy exist? Connection Science 18(4):403-417, 2006.

Calverley, David J. Android science and the animal rights movement: are there analogies. In Cognitive Sciences Society Workshop, Stresa, Italy, pp.127-136, 2005.

Chilvers, Jason. Reflexive Engagement? Actors, Learning, and Reflexivity in Public Dialogue on Science and Technology. Science Communication 35(3):283-310, 2013.

Chin, Matthew, Valerie Sims, Bryan Clark, Gabriel Lopez. Measuring individual differences in anthropomorphism toward machines and animals. In Proceedings of the 
Human Factors and Ergonomics Society Annual Meeting, vol 48, pp. 1252-1255, 2004.

Coeckelbergh, Mark. Robot rights? Towards a social-relational justification of moral consideration. Ethics and Information Technology 12(3):209-221, 2010.

Darling, Kate. Extending Legal Protection to Social Robots: The Effects of Anthropomorphism, Empathy, and Violent Behavior Towards Robotic Objects. In Ryan Calo, A. Michael Froomkin, and Ian Kerr (eds) Robot Law, Edward Elgar, 2016.

Delvaux, Mady (rapporteur). Draft Report with recommendations to the Commission on Civil Law Rules on Robotics. European Parliament Committee on Legal Affairs Report 2015/2103(INL), 2016.

Dick, Philip K. Do Androids Dream of Electric Sheep?, Doubleday, 1968.

Elbogen, Eric B., Sally C. Johnson, H. Ryan Wagner, Connor Sullivan, Casey T. Taft, and Jean C. Beckham. Violent behaviour and post-traumatic stress disorder in US Iraq and Afghanistan veterans. The British Journal of Psychiatry 204(5):368-375, 2014.

Epley, Nicholas, Adam Waytz and John T. Cacioppo. On seeing human: a threefactor theory of anthropomorphism. Psychological Review 114(4):864-886, 2007.

EPSRC. Principles of Robotics, Engineering and Physical Sciences Research Council, https://epsrc.ukri.org/research/ourportfolio/themes/engineering/activities/principlesofr obotics/ (last access April 2018), 2010.

Eyssel, Friederike, Dieta Kuchenbrandt, Simon Bobinger, Laura De Ruiter and Frank Hegel. "If you sound like me, you must be more human": On the interplay of robot and user features on human-robot acceptance and anthropomorphism. In Proceedings of the 7th annual ACM/IEEE International Conference on Human-Robot Interaction (HRI '12), pp. 125-126, 2012.

Ford, Martin. The Rise of the Robots: Technology and the Threat of Mass Unemployment, Oneworld Publications, 2015.

Forlizzi, Jodi and Carl DiSalvo. Service robots in the domestic environment: a study of the roomba vacuum in the home. In Proceedings of the 1st ACM SIGCHI/SIGART Conference on Human-Robot Interaction (HRI '06), pp. 258-265, 2006.

Future of Life Institute. Autonomous Weapons: An Open Letter From Ai \& Robotics Researchers, futureoflife.org/ai-open-letter/ (last access August 2017), 2015.

Fussell, Susan R., Sara Kiesler, Leslie D. Setlock and Victoria Yew. How people anthropomorphize robots. In Proceedings of the 3rd ACM/IEEE International Conference on Human-Robot Interaction (HRI 2008), pp. 145-152, 2008.

Garland, Alex (director). Ex Machina [Motion Picture], Universal Pictures, 2015. 
Gentner, Dedre and Kenneth D. Forbus. Computational models of analogy. WIREs Cognitive Science 2:266-276, 2011.

Gibson, William. Idoru, Viking Press, 1996.

Glas, Dylan F., Takashi Minato, Carlos T. Ishi, Tatsuya Kawahara, and Hiroshi Ishiguro. "ERICA: The ERATO Intelligent Conversational Android." Proceedings of the 25th IEEE International Symposium on Robot and Human Interactive Communication (RO-MAN), pp. 22-29, 2016.

Grodzinsky, Frances S., Keith W. Miller, and Marty J. Wolf. Developing automated deceptions and the impact on trust. Philosophy \& Technology 28(1):91-105, 2015.

Gunkel, David J. The Machine Question, MIT Press, 2012.

Gunkel, David J. A vindication of the rights of machines. Philosophy \& Technology 27(1):113-132, 2014.

Gunkel, David J. The other question: can and should robots have rights? Ethics and Information Technology, https://doi.org/10.1007/s10676-017-9442-4, 2017.

Hanson Robotics. Sophia. http://www.hansonrobotics.com/robot/sophia/ (last access April 2018), 2017.

Hauskeller, Michael. Automatic Sweethearts. In Mythologies of Transhumanism, Springer, pp. 181-199, 2016.

Hogan, Katharyn. Is the machine question the same question as the animal question? Ethics and Information Technology 19:29-38, 2017.

Holyoak, Keith J. and Kyunghee Koh. Surface and structural similarity in analogical transfer. Memory \& Cognition 15:332-340, 1987.

Johnson, Deborah G. and Mario Verdicchio. AI Anxiety. Journal of the Association for Information Science and Technology 68(9):2267-2270, 2017.

Jonze, Spike (director). Her [Motion Picture], Warner Bros., 2013.

Kant, Immanuel. Lectures on ethics, edited by Peter Heath and JB Schneewind, translated by Peter Heath, 1997.

Kelley, Richard, Enrique Schaerer, Micaela Gomez, and Monica Nicolescu. Liability in robotics: an international perspective on robots as animals. Advanced Robotics 24(13):1861-1871, 2010.

Kuehn, Johannes and Sami Haddadin. An Artificial Robot Nervous System To Teach Robots How To Feel Pain And Reflexively React To Potentially Damaging Contacts. IEEE Robotics and Automation Letters 2(1):72-79, 2017. 
Kurzweil, Raymond. The Singularity is Near: When Humans Transcend Biology, Penguin Books, 2005.

Latour, Bruno. Science in Action: How to Follow Scientists and Engineers Through Society, Harvard University Press, Cambridge, USA, 1987.

Levine, Sergey, Peter Pastor, Alex Krizhevsky and Deirdre Quillen. Learning HandEye Coordination for Robotic Grasping with Deep Learning and Large-Scale Data Collection. Google Preliminary Report available at https://arxiv.org/pdf/1603.02199v4.pdf, 2016.

Levy, David. Love and Sex with Robots, Harper Perennial, 2008.

Levy, David. The ethical treatment of artificially conscious robots. International Journal of Social Robotics 1(3):209-216, 2009.

Liang, Amy, Isabell Piroth, Hayley Robinson, Bruce MacDonald, Mark Fisher, Urs M. Nater, Nadine Skoluda, Elizabeth Broadbend. A Pilot Randomized Trial of a Companion Robot for People With Dementia Living in the Community. Journal of the American Medical Directors Association, doi.org/10.1016/j.jamda.2017.05.019 (last access August 2017), 2017.

Lin, Patrick, Keith Abney and George A. Bekey. Robot Ethics: The Ethical and Social Implications of Robotics, MIT Press, 2011.

MacLennan, Bruce. Cruelty to Robots? The Hard Problem of Robot Suffering. Proceedings of the 2013 Meeting of the International Association for Computing and Philosophy (IACAP), 2013.

MacManus, Deirdre, Roberto Rona, Hannah Dickson, Greta Somaini, Nicola Fear, and Simon Wessely. Aggressive and violent behavior among military personnel deployed to Iraq and Afghanistan: Prevalence and link with deployment and combat exposure. Epidemiologic Reviews 37(1):196-212, 2015.

Markey, Patrick M., Juliana E. French, and Charlotte N. Markey. Violent Movies and Severe Acts of Violence: Sensationalism Versus Science. Human Communication Research 41(2):155-173, 2014.

McNally, Phil and Sohail Inayatullah. The rights of robots: Technology, culture and law in the 21st century. Futures 20(2):119-136, 1988.

Metzinger, Thomas. Two Principles for Robot Ethics. In Eric Hilgendorf and JanPhilipp Günther (eds) Robotik und Gesetzgebung, 247-286, 2013.

Miller, Keith W. It's not nice to fool humans. IT professional 12(1):51-52, 2010.

Minsky, Marvin. Dr. Marvin Minsky - Facing the Future, www.youtube.com/watch?v=w9sujY8Xjro (last access June 2017), 2013.

Moore, Alan. V for Vendetta, DC Comics, 1989. 
Mori, Masahiro. The uncanny valley. Energy 7(4):33-35, 1970.

Mori, Masahiro, Karl F. MacDorman, Norri Kageki. The uncanny valley [from the field]. IEEE Robotics \& Automation Magazine 19(2):98-100, 2012.

Novaco, Raymond W., and Claude M. Chemtob. Violence associated with combatrelated posttraumatic stress disorder: The importance of anger. Psychological Trauma: Theory, Research, Practice, and Policy 7(5):485, 2015.

Owen, Richard, Jack Stilgoe, Phil Macnaghten, Mike Gorman, Erik Fisher, and Dave Guston. A Framework for Responsible Innovation. In Richard Owen, John Bessant and Maggie Heintz (eds) Responsible Innovation: Managing the Responsible Emergence of Science and Innovation in Society, John Wiley \& Sons, Ltd, Chichester, UK, 2013.

Parisi, Domenico. Future Robots: Towards a robotic science of human beings, John Benjamins Publishing, 2014.

Perkowitz, Sidney. Digital People: From Bionic Humans to Androids, Joseph Henry Press, 2004.

Robertson, Jennifer. Human Rights Vs. Robot Rights: Forecasts from Japan. Critical Asian Studies 46(4):571-598, 2014.

Ross, Brian H. Distinguishing types of superficial similarities: Different effects on the access and use of earlier problems. Journal of Experimental Psychology: Learning, Memory and Cognition 5:456-468, 1989.

Rysewyk, Simon van. Robot Pain. International Journal of Synthetic Emotions 4(2):22-33, 2014.

Schaerer, Enrique, Richard Kelley, and Monica Nicolescu. Robots as animals: A framework for liability and responsibility in human-robot interactions. In RO-MAN 2009-The 18th IEEE International Symposium on Robot and Human Interactive Communication, pp. 72-77, IEEE, 2009.

Schmidt, Colin T. A. Redesigning Man? In Pieter E. Vermaas, Peter Kroes, Andrew Light, Steven A. Moore (eds) Philosophy and Design: From Engineering to Architecture, Springer, pp. 209-216, 2008.

Sharkey, Noel, and Amanda Sharkey. The crying shame of robot nannies: an ethical appraisal. Interaction Studies 11(2):161-190, 2010.

Sharkey, Amanda, and Noel Sharkey. Granny and the robots: ethical issues in robot care for the elderly. Ethics and Information Technology 14(1):27-40, 2012.

Sharkey, Noel, Aimee van Wynsberghe, Scott Robbins and Eleanor Hancock. Our Sexual Future with Robots. The Hague, Netherlands: Foundation for Responsible Robotics. http://responsiblerobotics.org/wp-content/uploads/2017/07/FRR- 
Consultation-Report-Our-Sexual-Future-with-robots_Final.pdf (last access September 2017), 2017.

Solaiman, S. M. "Legal personality of robots, corporations, idols and chimpanzees: a quest for legitimacy." Artificial Intelligence and Law 25:155-179, 2017.

Spellman, Barbara A. and Keith J. Holyoak. Pragmatics in analogical mapping. Cognitive Psychology 31:307-346, 1996.

Spennemann, Dirk H. R. Of great apes and robots: Considering the future(s) of cultural heritage. Futures 39(7):861-877, 2007.

Sullins, John P. When is a robot a moral agent. International Review of Information Ethics 6(12):23-30, 2006.

Sullins, John P. When is a robot a moral agent? In Michael Anderson, Susan Leigh Anderson (eds) Machine Ethics, Cambridge University Press, Cambridge, UK, 2011. 